\title{
Maternal intake of high $n-6$ polyunsaturated fatty acid diet during pregnancy causes transgenerational increase in mammary cancer risk in mice
}

Nguyen M. Nguyen ${ }^{1}$, Fabia de Oliveira Andrade ${ }^{1}$, Lu Jin ${ }^{1}$, Xiyuan Zhang ${ }^{1}$, Madisa Macon ${ }^{1}$, M. Idalia Cruz', Carlos Benitez ${ }^{1}$, Bryan Wehrenberg ${ }^{2}$, Chao Yin ${ }^{1}$, Xiao Wang ${ }^{3}$, Jianhua Xuan ${ }^{3}$, Sonia de Assis ${ }^{1}$

and Leena Hilakivi-Clarke ${ }^{1 *}$

\begin{abstract}
Background: Maternal and paternal high-fat (HF) diet intake before and/or during pregnancy increases mammary cancer risk in several preclinical models. We studied if maternal consumption of a HF diet that began at a time when the fetal primordial germ cells travel to the genital ridge and start differentiating into germ cells would result in a transgenerational inheritance of increased mammary cancer risk.

Methods: Pregnant C57BL/6NTac mouse dams were fed either a control AIN93G or isocaloric HF diet composed of corn oil high in n-6 polyunsaturated fatty acids between gestational days 10 and 20. Offspring in subsequent F1-F3 generations were fed only the control diet.

Results: Mammary tumor incidence induced by 7,12-dimethylbenz[a]anthracene was significantly higher in F1 $(p<0.016)$ and F3 generation offspring of HF diet-fed dams $(p<0.040)$ than in the control offspring. Further, tumor latency was significantly shorter $(p<0.028)$ and burden higher $(p<0.027)$ in F1 generation HF offspring, and similar trends were seen in F3 generation HF offspring. RNA sequencing was done on normal mammary glands to identify signaling differences that may predispose to increased breast cancer risk by maternal HF intake. Analysis revealed 1587 and 4423 differentially expressed genes between HF and control offspring in F1 and F3 generations, respectively, of which 48 genes were similarly altered in both generations. Quantitative real-time polymerase chain reaction analysis validated 13 chosen up- and downregulated genes in F3 HF offspring, but only downregulated genes in F1 HF offspring. Ingenuity Pathway Analysis identified upregulation of Notch signaling as a key alteration in HF offspring. Further, knowledge-fused differential dependency network analysis identified ten node genes that in the HF offspring were uniquely connected to genes linked to increased cancer risk (ANKEF1, IGFBP6, SEMA5B), increased resistance to cancer treatments (SLC26A3), poor prognosis (ID4, JAM3, TBX2), and impaired anticancer immunity (EGR3, ZBP1).

Conclusions: We conclude that maternal HF diet intake during pregnancy induces a transgenerational increase in offspring mammary cancer risk in mice. The mechanisms of inheritance in the F3 generation may be different from the F1 generation because significantly more changes were seen in the transcriptome.
\end{abstract}

Keywords: Breast cancer, Transgenerational, n-6 Polyunsaturated fatty acids, Primordial germ cells, Maternal diet

\footnotetext{
* Correspondence: clarkel@georgetown.edu

'Department of Oncology, Georgetown University, Research Building, Room

E407, 3970 Reservoir Road, NW, Washington, DC 20057, USA

Full list of author information is available at the end of the article
} 


\section{Background}

With 1.7 million new cases in 2012, breast cancer is the most common cancer in women worldwide, and the incidence is projected to continue growing [1]. However, only $5-10 \%$ of breast cancers are attributed to an inherited genetic cause [2,3], and this leaves over $90 \%$ of breast cancers to be caused by some other factors. It is believed that environmental and lifestyle factors, such as diet, play a critical role in affecting breast cancer risk [4], and their effects on cancer susceptibility are likely to be mediated by epigenetic modifications [5].

Studies show that a Western dietary pattern, one that is higher in dietary fats, has been increasing around the world $[6,7]$ and may be linked to increased breast cancer risk [4]. Intake of dietary fats, especially saturated fatty acids and n- 6 polyunsaturated fatty acids ( $n-6$ PUFAs), has been climbing over the years [8], with intake of $n-3$ PUFAs declining [6]. A high-fat (HF) diet, especially one where n-6 PUFAs are high, increases estrogenic activities $[9,10]$ and is inflammatory $[11,12]$, which can stimulate breast cancer growth. Our laboratory and others have shown in animal models that maternal exposure to a HF diet during pregnancy increases female offspring's mammary cancer risk $[9,13-16]$. In addition, we found that maternal intake of an n-6 PUFA HF diet consumed before and during pregnancy increased mammary cancer risk of daughters (F1) and granddaughters (F2), but not of greatgranddaughters (F3) [13], indicating multigenerational but not transgenerational inheritance [17]. Multigenerational inheritance of a trait caused by maternal exposures during pregnancy, such as an increased susceptibility to breast cancer, is seen in F1 and/or F2 but not in F3 generation offspring. If the trait is also seen in F3 generation, inheritance is transgenerational [18].

The lack of transgenerational inheritance in the offspring of HF diet-fed dams was puzzling because previously we and others discovered that several different exposures during pregnancy led to a transgenerational increase in mammary cancer risk and other adverse health effects in F1, F2, and F3 generations. Specifically, transgenerational inheritance of adult-onset diseases and abnormalities, such as mammary cancer [13], prostate disease [19], obesity [20], and autism-like phenotype [21], has been found in offspring of dams that were exposed to compounds such as ethinylestradiol [13], vinclozolin [19], dichlorodiphenyltrichloroethane (DDT) [20], and valproic acid [21] during pregnancy. Common to all these studies was that the maternal exposure took place after fetal implantation, whereas a HF diet that resulted in a multigenerational increase in mammary cancer risk was started before conception [13].

Mammals undergo two rounds of global DNA demethylation and remethylation during the embryonic and fetal periods [22]. It is possible that in our previous study, the reason why we did not see true transgenerational inheritance of increased breast cancer risk was due to introducing the HF exposure throughout the first round of DNA methylation erasure in the zygote and the subsequent remethylation, as well as during the second round of methylation erasure, which took place in primordial germ cells (PGCs), and the second remethylation, which reestablished epigenetic marks on mature germ cells [23]. Because PGCs originate from the posterior endoderm of the blastocyst, the second round of epigenetic programming may have been affected by the presence of the HF diet during the first round. To test a possibility that maternal HF intake induces a transgenerational inheritance of mammary cancer risk, we timed the HF exposure to start on gestational day (GD) 10. Pregnant women consume more dietary fats than nonpregnant women, and the increase takes place between the first and second trimesters [24, 25].

Our study shows that maternal intake of a HF diet between GDs 10 and 20 caused a transgenerational increase in mammary cancer risk tumors without any other intervening dietary exposures. Results from RNA sequencing (RNA-seq) analysis suggested that there was a difference in how mammary cancer risk was affected in F1 and F3 generation offspring by maternal HF intake (i.e., whether the exposure directly affected fetal somatic cells or the germline).

\section{Methods \\ Breeding and dietary exposure}

Male and female C57BL/6NTac mice were obtained from Taconic Biosciences (Germantown, NY, USA) and housed in standard rodent housing at constant temperature and humidity and with a 12-h/12-h light/ dark cycle at Georgetown University's Department of Comparative Medicine, in accordance with all institutional and federal regulations.

To generate F1 offspring (Fig. 1a), 7-week-old mice were mated by housing two females together with one male per cage. Upon mating, mice were randomly divided into two groups and fed a control AIN93G diet until pregnancy was verified by the presence of a mucus copulatory plug in the vaginal opening (GD 0). From GDs 10 to 20, the pregnant dams (F0) were divided into two groups and fed either a HF diet containing $41.1 \%$ energy from fat, $38.9 \% \mathrm{kcal}$ of corn oil (CO), and $2.2 \% \mathrm{kcal}$ of soybean oil (SBO) (Additional file 1: Table S1), or they were continued on the control diet $(16.0 \%$ energy from fat, $13.7 \% \mathrm{kcal}$ of CO, $2.2 \% \mathrm{kcal}$ of SBO) (Additional file 1: Table S1). Because the length of pregnancy in these mice is 19-21 days, the HF exposure took place during the second and third mouse trimesters. The HF diet was made isocaloric with the control diet by replacing some carbohydrates with non-energy-containing cellulose (fiber). $\mathrm{CO}$ 


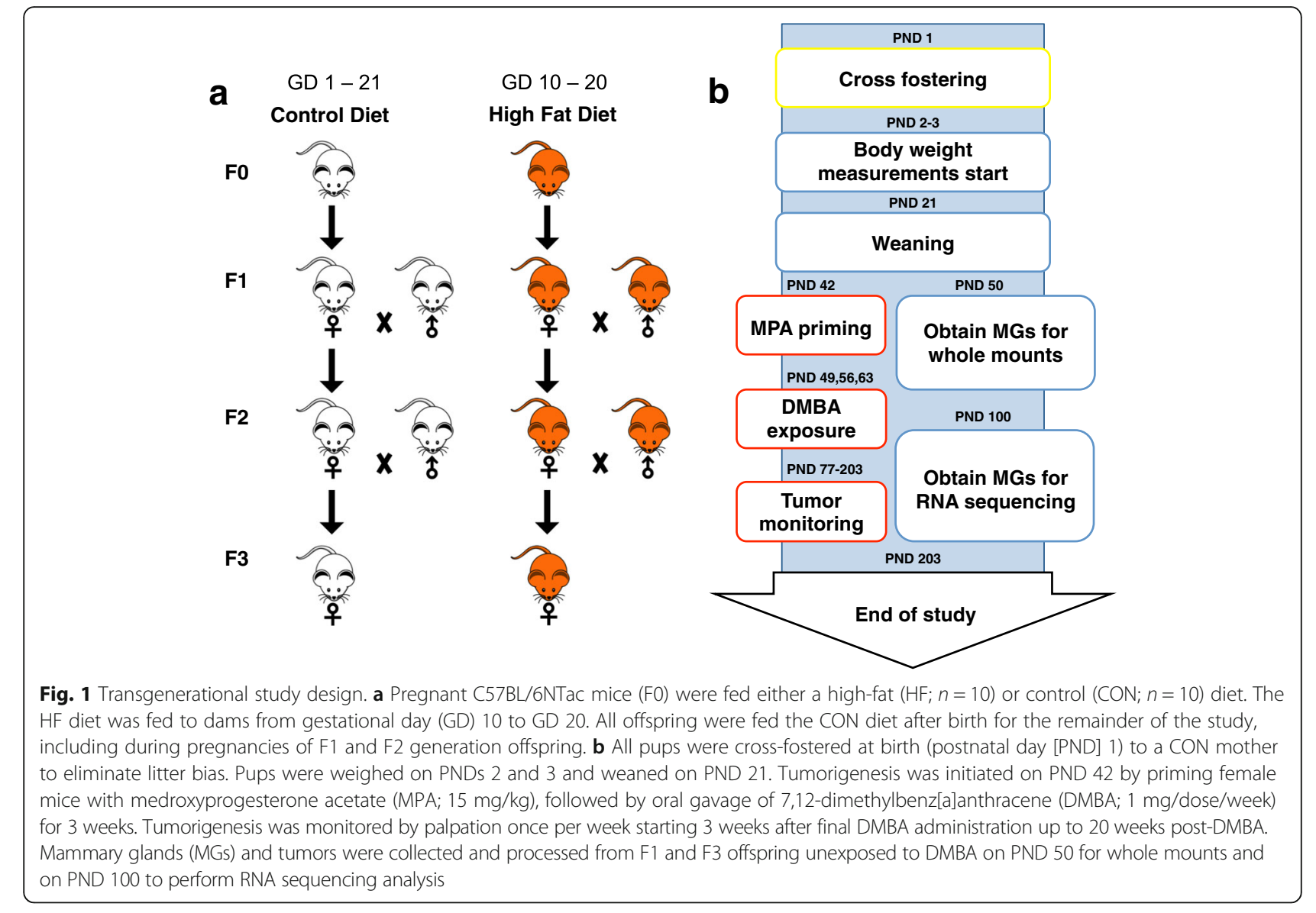

contains high levels of n-6 PUFAs. Both diets contained $10 \mathrm{~g} / \mathrm{kg}$ SBO to ensure that pregnant dams received sufficient levels of n-3 PUFAs. All subsequent generations (F1-F3) were fed only the control diet.

To obtain F3 generation offspring, female F1 generation offspring of dams fed a HF diet during pregnancy were mated to F1 HF diet-fed males, respectively. No sibling matings were performed. The control group was mated similarly. Pregnant F1 dams of both groups were fed the $\mathrm{CON}$ diet. These steps were repeated with F2 offspring to obtain the F3 generation. The F2 generation was used for breeding purposes only; that is, no experiments were performed with or tissues collected from them.

\section{Mammary tumorigenesis}

To induce mammary tumors (Fig. 1b), female C57BL6/ NTac mice ( $n=30$ for F1 control, $n=30$ for F1 HF; $n=$ 20 for F3 control, and $n=25$ for F3 HF offspring) were first primed with $15 \mathrm{mg} / \mathrm{kg}$ of medroxyprogesterone acetate (MPA; Greenstone, Peapack, NJ, USA) at postnatal day (PND) 42. One milligram of 7,12-dimethylbenz[a] anthracene (DMBA; Sigma-Aldrich, St. Louis, MO, USA) in $0.1 \mathrm{ml}$ of $\mathrm{CO}$ was administered by oral gavage on PNDs 49, 56, and 63. After the last DMBA dose, tumor development was monitored by palpation once weekly for 20 weeks. If tumors were detected, their sizes were measured by calipers. The following endpoints were determined: incidence (number of mice with tumors), latency (time to first tumor), multiplicity (number of tumors per mouse), and burden (total tumor volume per mouse). The overall health of mice was monitored daily. A mouse was killed prior to the end of the tumormonitoring period if it lost a significant amount of weight or if the tumor reached $10 \%$ of the mouse's body weight. Mice killed for health reasons not pertaining to the study were excluded from the analysis. Tumor histopathology was assessed by a certified pathologist.

Kaplan-Meier survival curves were used to assess differences in tumor incidence between groups, followed by log-rank tests. Tumor latency and multiplicity differences were assessed by $t$ test. Difference in tumor burden was assessed by repeated measures analysis of variance (ANOVA).

\section{Tissue collection}

Fourth mammary glands were obtained on PND 50 and PND 100 from offspring not exposed to the carcinogen to assess changes in mammary gland morphology and 
transcriptome, respectively (Fig. 1b). At the end of the tumor-monitoring period, offspring in F1 and F3 generations were killed, and whole blood was collected via cardiac puncture and placed into serum gel separator tubes. We then collected mammary tumors, resected a portion for formalin-fixed, paraffin embedding, and flash-froze the remaining tissues in cryotubes in liquid nitrogen.

\section{Terminal end buds}

Whole mounts were prepared according to an established protocol [26]. For that purpose, the left fourth abdominal mammary glands were stretched onto a slide, fixed, and stained with carmine aluminum solution. Slides were examined blindly under a microscope to determine the total number of terminal end buds (TEBs). These are the structures that give rise to malignant mammary tumors in mice and rats [27] and to similar structures in humans, called terminal ductal lobular units, which also are the sites of most breast cancers [28]. Differences in TEB numbers between the offspring of dams fed the HF or control diet were analyzed by $t$ tests in both generations separately.

\section{RNA sequencing}

To identify differentially expressed genes (DEGs) between offspring of dams exposed to the HF or control diet during pregnancy, RNA-seq was performed. Total RNA was extracted from the right fourth mammary glands obtained on PND 100 from F1 and F3 generation offspring that were not treated with DMBA by using the RNeasy Lipid Tissue Mini Kit (QIAGEN Sciences, Germantown, MD, USA) with on-column DNase digestion (QIAGEN Sciences) per the manufacturer's protocol. The concentration and purity of the extracted RNA were determined by using a NanoDrop 1000 spectrophotometer (Thermo Scientific, Wilmington, DE, USA). The quality of the samples was assessed using a 2100 Bioanalyzer (Agilent Technologies, Santa Clara, CA, USA) for RNA integrity number $(>7.0)$ and concentration (minimum $70 \mathrm{ng} / \mu \mathrm{l}$ ). RNA-seq was performed by GENEWIZ (South Plainfield, NJ, USA). The HiSeq 2500 platform (Illumina, San Diego, CA, USA) was used for RNA-seq in a $1 \times 50$ bp single-read configuration in rapid run mode, with a total of at least 120 million reads per lane over 5 lanes with a read depth of at least 10 million reads per sample. A total of five control and three HF offspring in the F1 generation and four control and five HF offspring in the F3 generation were used for RNA-seq analysis.

Rsem was used to quantify transcript abundance using the mouse genome Mus musculus as a reference. With a $p$ value cutoff of 0.05 , we obtained 5620 DEGs for further analysis. We then selected those 48 DEGs that were seen in both F1 and F3 generation offspring, with the direction (up- or downregulation) of differential expression being similar in the two generations, and performed Ingenuity Pathway Analysis (IPA; QIAGEN Bioinformatics, Redwood City, CA, USA) to assess function of the DEGs. Knowledge-fused differential dependency network (KDDN) analysis was also performed on the 48 DEGs to assess transcriptional gene interaction unique to either control or HF offspring [29].

\section{Validation of RNA-seq data by quantitative real-time polymerase chain reaction}

Of the 48 DEGs, we attempted to validate the expression of 13 genes that were selected on the basis of their reported association with breast cancer. RNA was extracted from the right fourth mammary glands of six control and six HF offspring of F1 generation and also of six control and six HF offspring of F3 generation using the RNeasy Lipid Tissue Mini Kit per the manufacturer's protocol. These mice included those used for RNA-seq. The concentration, purity, and quality of RNA samples were assessed as described above. A quantity of $2 \mu \mathrm{g}$ of RNA per sample was used to generate complementary DNA (cDNA) via reverse transcription using the High-Capacity cDNA Reverse Transcription Kit (Applied Biosystems, Foster City, CA, USA) and was run on a PTC-100 thermal cycler (Bio-Rad Laboratories, Hercules, CA, USA). Product cDNA was brought to a working concentration of $5 \mathrm{ng} / \mu \mathrm{l}$ and mixed with ABsolute QPCR, SYBR Green, ROX mix (Thermo Fisher Scientific, Waltham, MA, USA) and gene-specific forward and reverse primers. Primers used in quantitative polymerase chain reaction (qPCR) analysis were designed using IDT tool primer design (Integrated DNA Technologies, Coralville, IA, USA) (primer sequence provided in Additional file 2: Table S2). Real-time qPCR was carried out using a 7900HT Real-Time PCR system (Applied Biosystems). Expression of target genes was calculated by the relative standard curve method normalized to the housekeeping gene glyceraldehyde 3-phosphate dehydrogenase $(G A P D H)$. Statistical differences between the control and F1 HF and F3 HF groups were assessed by one-way ANOVA.

\section{Results \\ Effect of maternal HF intake on offspring mammary tumorigenesis}

Maternal exposure to a HF diet during pregnancy did not affect the distribution of benign versus malignant mammary tumors among the offspring. Malignant mammary tumors were those that were either papillary or tubular adenocarcinomas or mammary carcinomas. Among the control offspring, $58.3 \%$ and $64.3 \%$ were malignant in the F1 and F3 generations, respectively (Additional file 3: Figure S1a), compared with $66.0 \%$ and $77.3 \%$ in the $\mathrm{F} 1$ and F3 generation HF offspring (Additional file 3: Figure S1b). 
When we assessed differences in tumor incidence, we observed that only those with a malignant phenotype were included in the analysis. Female offspring exposed to the HF diet through a pregnant dam exhibited increased tumor incidence in F1 (Fig. 2a) $(p<0.016)$ and F3 (Fig. 2b) $(p<0.040)$ generations compared with control offspring. Mammary tumor burden was also increased in the F1 generation (Fig. 2c) $(p<0.027)$, but the increase failed to reach statistical significance in the F3 generation (Fig. 2d) $(p<0.242)$.

Maternal HF exposure during pregnancy induced earlier onset of mammary cancer in F1 generation (Fig. 2e) $(p<0.028)$ and had a similar trend in F3 generation offspring (Fig. 2f) $(p<0.110)$. Mammary tumor multiplicity was unaffected by maternal HF exposure (Additional file 4: Figure S2).

\section{Offspring mammary gland morphology}

Mammary gland morphology was assessed using whole mounts obtained from female offspring at PND 50. The number of TEBs (indicated by the arrows in Fig. 3a) was counted and found to be significantly higher in HF offspring for both F1 (Fig. 3b) $(p<0.035)$ and F3 generations (Fig. 3c) $(p<0.023)$.

\section{Differentially expressed genes in mammary glands of $\mathrm{HF}$ diet-exposed offspring}

To elucidate the potential differences in mammary cancer risk in the F1 and F3 generation offspring of HF diet-fed dams, RNA-seq analysis was performed on normal mammary glands. In the F1 generation, 1587 DEGs were identified, and in the F3 generation, 4423 DEGs were seen (Fig. 4a). Of these, 390 were the same genes in both F1 and F3 HF offspring. However, only 48 of the DEGs were altered in the same direction (up- or downregulated) in both generations. Heat maps of these genes are shown in Fig. 4b and c and Additional file 5: Table S3.

IPA indicated that the top pathways that were different between HF and control offspring in both F1 and F3 generations were related to vitamin $\mathrm{D}$ receptor/retinoid $\mathrm{X}$ receptor (VDR/RXR) activation, phosphatase and tensin homolog (PTEN) signaling, farnesoid X receptor/ RXR (FXR/RXR) activation, hereditary breast cancer signaling, and Notch signaling (Additional file 6: Table S4). Top upstream regulators of these pathways were interferon regulatory factors 3 and 7 (IRF3 and IRF7, respectively) linked to interferon and macrophage regulation [30], and delta like canonical ligand 3 (DLL3), Jagged 1 (JAG1), and mesogenin 1 (MSGN1), which all are linked to Notch signaling [31, 32] (Additional file 6: Table S4). Top diseases and biofunctions involved development, cellular functions, cancer and tumor morphologies, and inflammatory responses (Additional file 7: Table S5).

KDDN analysis (Fig. 4d and e, Table 1) performed to identify unique gene signaling interactions present in either the HF or control offspring highlighted the following ten genes as nodes: ALG6, DPF3, GRHL3, MAGIX, $M T-T S 2$, and OPTC (full gene names provided in Abbreviations section below; upregulated in HF offspring), as well as AKR1C14, SLC5A3, SLC6A2, and ZBP1 (downregulated in HF offspring). These nodes had different signaling connections in $\mathrm{HF}$ and $\mathrm{CON}$ offspring. The node genes in the offspring of HF diet-fed dams were linked to changes in genes that are indicative of

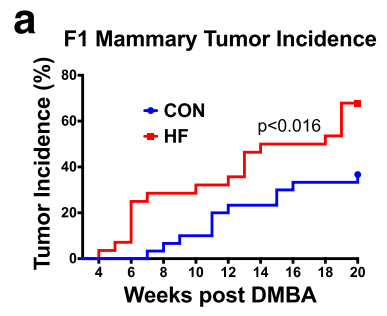

b

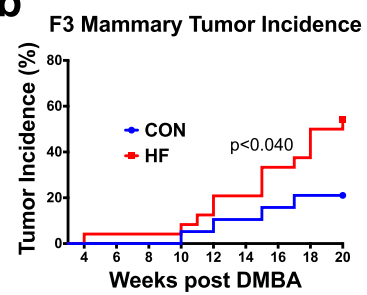

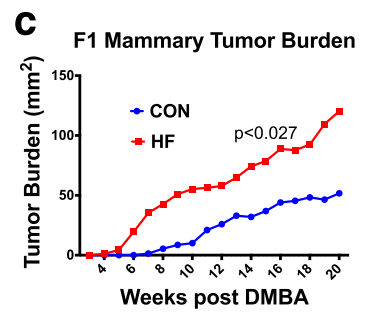

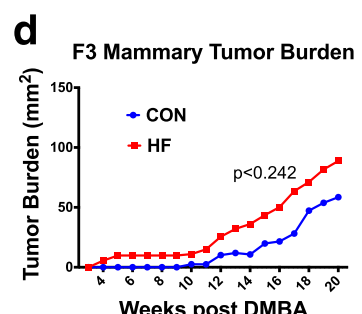

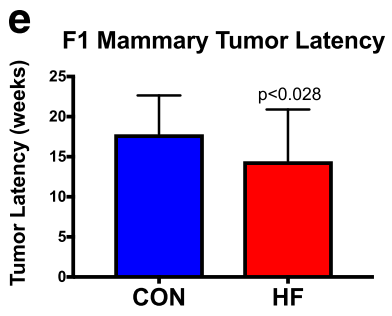

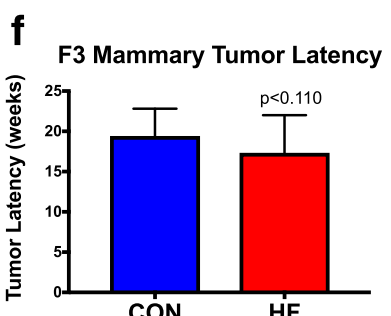

Fig. 2 Transgenerational effect of maternal control (CON) or high-fat (HF) diet on offspring mammary tumorigenesis. Differences in mammary tumor incidence in (a) F1 ( $p<0.016$; CON, $n=30$ mice; HF, $n=28$ mice) and (b) F3 ( $p<0.040 ; C O N, n=19$ mice; HF, $n=24$ mice) generation female offspring of dams fed either CON or HF diet during pregnancy. Differences in mammary tumor burden in (c) F1 $(p<0.027)$ and (d) F3 $(p<0.242)$ generation female offspring. Differences in mammary tumor latency in (e) F1 $(p<0.028)$ and (f) F3 $(p<0.110)$ generation female offspring. Mean \pm SEM data are shown in $\mathbf{c}-\mathbf{f}$. TEB Terminal end bud 
a

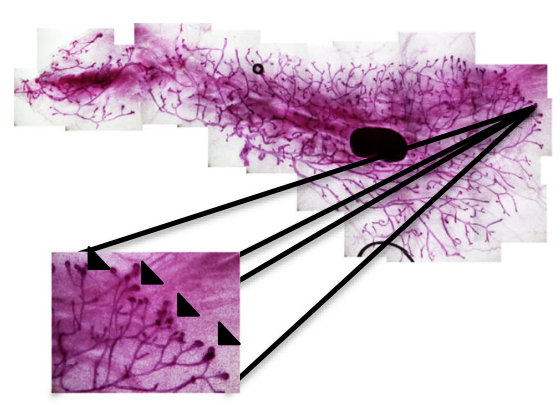

b

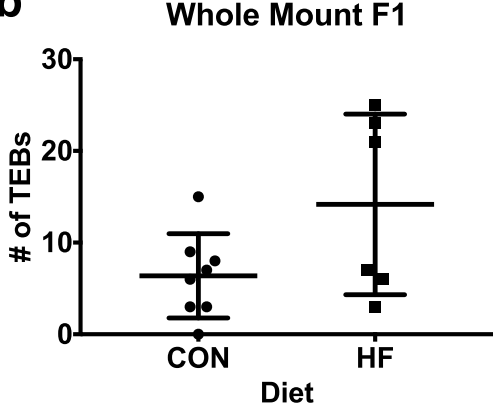

C

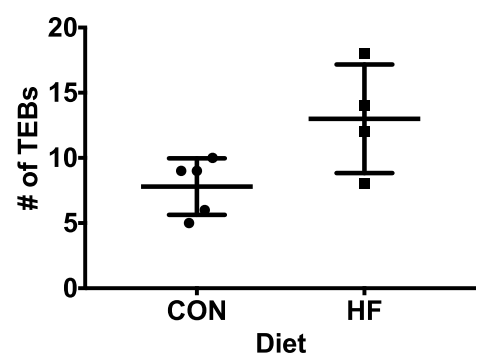

Fig. 3 Effect of maternal control (CON) or high-fat (HF) diet exposure on offspring mammary gland development. a The left fourth mammary glands were obtained on postnatal day 50 for whole mounts. Terminal end buds, structures in the enlarged image indicated by the arrows, were counted for (b) F1 ( $p<0.035 ; n=8$ for HF and $n=6$ for CON) and (c) F3 ( $p<0.023 ; n=5$ for HF and $n=4$ for CON). Mean \pm SEM data are shown. DMBA 7,12-Dimethylbenz[a]anthracene

increased cancer risk (downregulation of ANKEF1, IGFBP6; upregulation of $S E M A 5 B)$, increased resistance to cancer treatments (upregulation of EGR3, SLC26A3), poor cancer prognosis (upregulation of ID4, JAM3, TBX2), increased risk of metastasis (upregulation of GPCPD1) and impaired anticancer immune response (downregulation of $Z B P 1$, upregulation of $E G R 3$ ) (Additional file 8: reference table). In contrast, the ten node genes in the mammary glands of the offspring of dams fed the CON diet were linked to changes in the expression of genes indicative of reduced cancer risk (downregulation of DPF3, SNORA41) and improved immune functions (upregulation of ZBP1, ZFP683; downregulation of EGR3). These differences potentially play a regulatory role in causing a Differentially expressed genes

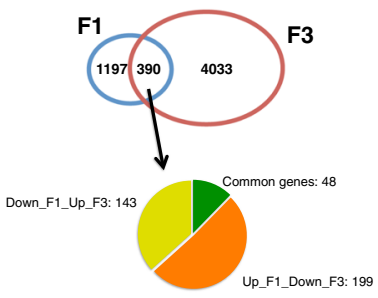

C

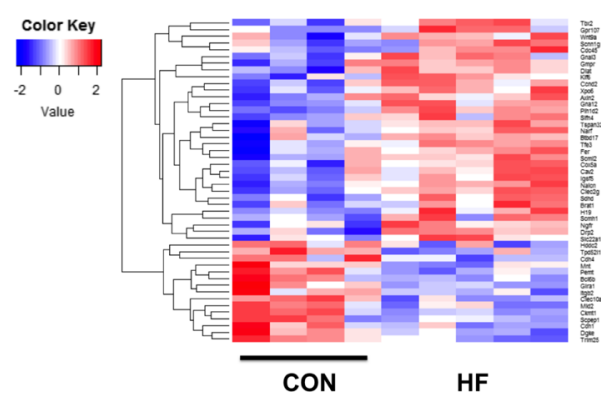

b

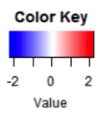

Value

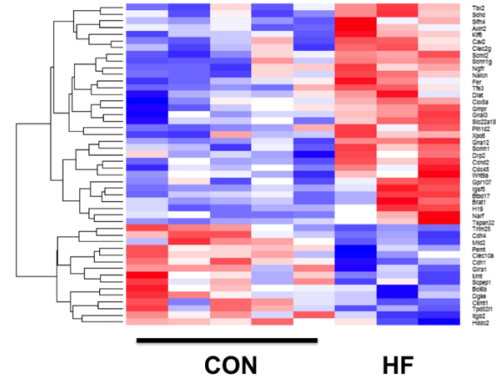

d

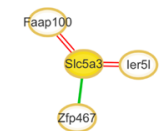

e

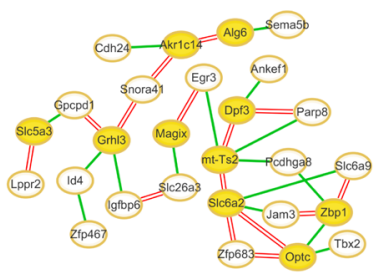

Fig. 4 Differentially expressed genes (DEGs) in mammary glands of F1 and F3 generation offspring of dams fed either control (CON) or high-fat (HF) diet during pregnancy. a RNA-sequencing analysis identified 1587 DEGs in F1 and 4423 DEGs in F3 generation mammary glands obtained on postnatal day 100 from mice not exposed to 7,12-dimethylbenz[a]anthracene ( $n=5 \mathrm{CON}$ and $n=3 \mathrm{HF}$ offspring in F1 generation, and $n=4$ CON and $n=5 \mathrm{HF}$ offspring in F3 generation). A total of 390 common DEGs were found in the F1 and F3 generations, with 48 regulated in the same direction in both generations. Heat map of common 48 DEGs in (b) F1 mammary glands and (c) F3 mammary glands. Knowledge-fused differential dependency networks cluster map of nodes uniquely connected to different sets of genes in HF or CON offspring in (d) F1 or (e) F3 generation. Yellow ovals indicate nodes. Single-lined green connections indicate gene interactions in HF offspring. Double-lined red connections indicate gene interaction in CON offspring 
Table 1 Upregulated and downregulated node genes in normal mammary glands of F1 and F3 generation mouse offspring of dams fed a high-fat diet and their unique signaling connections identified via knowledge-fused differential dependency network analysis ${ }^{a}$

\begin{tabular}{|c|c|c|}
\hline Upregulated gene (function) & $\begin{array}{l}\text { Connections Consequence } \\
\text { in HF } \\
\text { offspring }\end{array}$ & $\begin{array}{l}\text { Connections Consequence } \\
\text { in control } \\
\text { offspring }\end{array}$ \\
\hline
\end{tabular}

Alg6 Encodes glucosyltransferase, critical role in N-glycosylation [1]

Sema5b个

O-glycosylation of thrombospondin type 1 repeat domain-containing proteins; implicated in clear cell renal cell carcinoma [17] as well as other age-related diseases, including cancer [18]

Dpf3 Chromatin remodeling; associated with increased breast cancer risk, tumor size, earlier onset of disease, and lymph node metastases [2]; also implicated in chronic lymphocytic leukemia through STAT5 regulation [3]

Grh/3 Regulator of developmental processes; overexpression increases cell migration and invasion by downregulating E-cadherin [4]; strongly implicated in breast cancer [5]

Ankef1 $\downarrow$

Calcium ion binding; found as significant predictor of prostate cancer in GWAS SNPs study [19]

Akr1c14个

3a-Hydroxysteroid dehydrogenase enzyme; catalyzes conversion of potent testosterones into less potent forms [8]

Upregulated in acute leukemia [20]; catalyzes posttranslational modification of protein by addition of ADP-ribose moieties; contributes to survival of injured proliferating cells [21]

Mt-Ts2 $\uparrow \quad$ RNA gene affiliated with noncoding RNA class; possible association with mitochondrial disorders [9]

Id $4 \uparrow \quad$ A lineage-dependent proto-oncogene that is overexpressed and amplified in a subset of basal-like breast cancers and confers a poor prognosis [22]; suppresses BRCA1 [23, 24]

lgfbp6 $\downarrow$

Magix Function not known

$\operatorname{Slc} 26 \mathrm{a} 3 \uparrow$

GF-1-binding protein; lower expression in malignant breast cancer than in benign tumors [26]; also overexpressed in lung cancer and rhabdomyosarcomas [27]

Glycoprotein, a marker of chemoresistance in $\mathrm{ER}^{+}$breast cancer [30]

Optc Extracellular matrix glycoprotein [6], found translocated to nucleus in chronic lymphocytic leukemia cells [7]

Downregulated gene (function)

Akr1c14 3a-hydroxysteroid dehydrogenase enzyme; catalyzes conversion of potent testosterones into less potent forms [8]

Mt-Ts2 RNA gene affiliated with noncoding RNA class; possible association with mitochondrial disorders [9]
Regulator of developmental processes; increased expression predicts poor prognosis for many cancer types, including head and neck cancers [35], gastric cancer [36], and breast cancer [37]; contributes to drug resistance in breast cancer [38]

Zbp1 $\downarrow \quad$ Activator of innate immune response $[14,15]$ with potential to promote effective antitumor $\mathrm{CD}^{+}{ }^{+} \mathrm{T}$-cell immunity [16]

\section{Connections Consequence} in $\mathrm{HF}$

Cdh $24 \uparrow$ Induces cell adhesion; mutation target in cancers with microsatellite instability, particularly gastric and colorectal

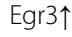

Mediates E2-induced breast cancer metastasis [31]; upregulated in endocrineresistant breast cancers [32]; increases aromatase in breast tissue [33]; maintains tumor immunosuppression [34]

Parp8 $\quad \quad$ Upregulated in acute leukemia [20]; catalyzes posttranslational modification of protein by addition of ADP-ribose moieties, contributes to survival of injured proliferating cells [21] cancers [40]
Gpcpd1 $\downarrow \quad$ Downregulation reduces migration capacity of tumor cells and is a prognostic indicator of good outcome in endometrial and ovarian cancers [25]

Snora41 $\downarrow \quad$ Long noncoding RNA linked to embryonic stem cell differentiation [28]; upregulated in lung cancer [29]

Egr3 $\downarrow$

Mediates E2-induced breast cancer metastasis [31]; upregulated in increases aromatase in breast tissue [33]; maintains tumor immunosuppression [34]

Slc6a2 $\uparrow$ Induces norepinephrine uptake [11] upregulation lowers risk of pancreatic ductal adenocarcinoma [12] and nonsmall-cell lung cancer [13]

Zfp683个

Essential for formation of mature thymic natural killer cells [39]

Connections Consequence in $\mathrm{CON}$

Alg6 $\downarrow \quad$ Encodes glucosyltransferase, critical role in N-glycosylation [1]

Snora41 $\downarrow$ Long noncoding RNA linked to embryonic stem cell differentiation [28]; upregulated in lung cancer [29]

Dpf3 $\downarrow \quad$ Polymorphisms in $5^{\prime}$ region associated with increased breast cancer development

Zbp1 $1 \quad$ Activator of innate immune response $[14,15]$ with potential to promote effective antitumor CD8 ${ }^{+}$T-lymphocyte immunity [16] endocrine-resistant breast cancers [32]; 
Table 1 Upregulated and downregulated node genes in normal mammary glands of F1 and F3 generation mouse offspring of dams fed a high-fat diet and their unique signaling connections identified via knowledge-fused differential dependency network analysis ${ }^{a}$ (Continued)

\begin{tabular}{|c|c|c|c|c|}
\hline & Pcdhga8 $\uparrow$ & Establishes cell-cell connections [41] & & \\
\hline \multirow[t]{3}{*}{$\begin{array}{l}\text { S/c5a3 Transporter; downregulation } \\
\text { impairs cellular functions by causing } \\
\text { accumulation of myoinositol, } \\
\text { downregulated in type } 2 \text { diabetes [10] }\end{array}$} & Gpcpd1个 & $\begin{array}{l}\text { Decreased expression increases the } \\
\text { migration capacity of tumor cells and } \\
\text { worsens prognosis of endometrial and } \\
\text { ovarian cancers [25] }\end{array}$ & $|e r 5| \downarrow$ & $\begin{array}{l}\text { Immediate early response gene; may } \\
\text { mediate actions on PUFAs [45] }\end{array}$ \\
\hline & Zfp467^ & $\begin{array}{l}\text { Promotes adipocyte differentiation and } \\
\text { inhibits osteoblast differentiation [43] }\end{array}$ & Faap100』 & Required for E3 ligase function [44] \\
\hline & & & Lppr $2 \downarrow$ & Peptide-ligand binding, GPCR signaling [42] \\
\hline \multirow[t]{3}{*}{$\begin{array}{l}\text { Slc6a2 Norepinephrine uptake } \\
\text { regulator [11]; downregulation } \\
\text { increases risk of pancreatic ductal } \\
\text { adenocarcinoma [12] and non- } \\
\text { small-cell lung cancer [13] }\end{array}$} & $\operatorname{Jam} 3 \uparrow$ & $\begin{array}{l}\text { Cell-cell adhesion; upregulation } \\
\text { associated with poor prognosis for } \\
\text { non-small-cell lung cancer [46] and } \\
\text { gastric cancer [47]; promotes ovarian } \\
\text { tumors in mice [48] }\end{array}$ & Optc $\downarrow$ & $\begin{array}{l}\text { Extracellular matrix glycoprotein [6], } \\
\text { found translocated to nucleus in } \\
\text { chronic lymphocytic leukemia cells [7] }\end{array}$ \\
\hline & Slc6a9个 & $\begin{array}{l}\text { Transporter that inhibits glycine } \\
\text { signaling [49]; solute carrier } 6 \text { family is } \\
\text { implicated in many cancers [50] }\end{array}$ & Zbp $1 \uparrow$ & $\begin{array}{l}\text { Activator of innate immune response [14, } \\
\text { 15] with potential to promote effective } \\
\text { antitumor } C D 8^{+} \text {-cell immunity [16] }\end{array}$ \\
\hline & & & Zfp683个 & $\begin{array}{l}\text { Essential for formation of mature } \\
\text { thymic natural killer cells [39] }\end{array}$ \\
\hline \multirow[t]{4}{*}{$\begin{array}{l}\text { Zbp1 Activator of innate immune } \\
\text { response }[14,15] \text { with potential to } \\
\text { promote effective antitumor CD8 } 8^{+} \\
\text {T-cell immunity }[16]\end{array}$} & Pcdhga8^ & Establishes cell-cell connections [41] & Slc6a2 $\uparrow$ & $\begin{array}{l}\text { Induces norepinephrine uptake [11]; } \\
\text { upregulation lowers risk of pancreatic } \\
\text { ductal adenocarcinoma [12] and } \\
\text { non-small-cell lung cancer [13] }\end{array}$ \\
\hline & Optc $\uparrow$ & $\begin{array}{l}\text { Extracellular matrix glycoprotein [6] } \\
\text { found translocated to nucleus in } \\
\text { chronic lymphocytic leukemia cells [7] }\end{array}$ & $\operatorname{Jam} 3 \downarrow$ & $\begin{array}{l}\text { Cell-cell adhesion; downregulation } \\
\text { linked to better prognosis for non- } \\
\text { small-cell lung cancer [46] and gastric } \\
\text { cancer [47]; upregulation promotes } \\
\text { ovarian tumor in murine model [48] }\end{array}$ \\
\hline & & & Mt-Ts2个 & $\begin{array}{l}\text { RNA gene, affiliated with noncoding } \\
\text { RNA class, possible association with } \\
\text { mitochondrial disorders [9] }\end{array}$ \\
\hline & & & Slc6a9 $\downarrow$ & $\begin{array}{l}\text { Transporter that inhibits glycine } \\
\text { signaling [49]; solute carrier } 6 \text { family is } \\
\text { implicated in many cancers [50] }\end{array}$ \\
\hline
\end{tabular}

Abbreviations: Akr1c14 Aldo-keto reductase family 1, member C14, Alg6 a-1,3-Glucosyltransferase, Ankef1 Ankyrin repeat and EF-hand domain containing 1, Dpf3 Double PHD fingers 3, E2 17- $\beta$-Estradiol, Egr3 Early growth response 3, ER Estrogen receptor, Gpcpd1 Glycerophosphocholine phosphodiesterase 1, GPCR G protein-coupled receptor, Grh/3 Grainyhead like transcription factor 3, GWAS Genome-wide association study, HF High fat, Id4 DNA-binding protein inhibitor ID-4, IGF-1 Insulin-like growth factor 1, Igfbp6 Insulin-like growth factor binding protein 6, Jam3 Junctional adhesion molecule 3, Magix MAGI family member, X-linked, Mt-Ts2 Mitochondrially encoded transfer RNA serine 2 (AGU/C), Optc Opticin, Pcdhga8 Protocadherin gamma subfamily A, 8, PUFA Polyunsaturated fatty acid, Sema5b Semaphorin 5B, Slc26a3 Solute carrier family 26 member 3, Slc5a3 Solute carrier family 5 member 3, S/c6a2 Solute carrier family 6 member 2, Snora41 Small nucleolar RNA, H/ACA box 41, SNP Single-nucleotide polymorphism, STAT5 Signal transducer and activator of transcription 5, Tbx2 T-box 2, Zbp1 Z-DNA binding protein 1, Zfp683 Zinc finger protein 683

${ }^{\text {a }}$ Connection of the node genes in control offspring are also shown

transgenerational inheritance of increased mammary cancer risk in the offspring of dams fed the HF diet during pregnancy.

\section{Verification of differential gene expression}

Quantitative real-time polymerase chain reaction (qRTPCR) analysis indicated that all 13 genes differentially expressed in the RNA-seq dataset between offspring of $\mathrm{HF}$ and control diet-fed dams were validated in the F3 generation (Fig. 5). However, among the F1 generation, none of the eight upregulated genes were validated: AKT2, EGR3, HES1, ID4, JAM3, PCDHGA8, SLC26A10, and TBX2 (Fig. 5a-h). Of the five downregulated genes (IGFBP6, OAS3a, P21, SLFN1, and ZBP1) (Fig. 5i-m), four were significantly and one was nonsignificantly downregulated in both the F1 (OAS3a was not significant) and F3 (IGFBP6 was not significant) generations.

\section{Discussion}

We found that maternal intake of a HF n-6 PUFA diet, starting on GD 10 during the second half of pregnancy and continuing until the end of pregnancy, increased estrogen receptor-positive $\left(\mathrm{ER}^{+}\right)$mammary cancer risk in F1 and F3 generation mouse offspring. Transgenerational inheritance likely requires that epigenetic changes induced by maternal exposures during pregnancy persist in germ cells. This possibility has been debatable because when F1 generation germ cells become fertilized, parental DNA 


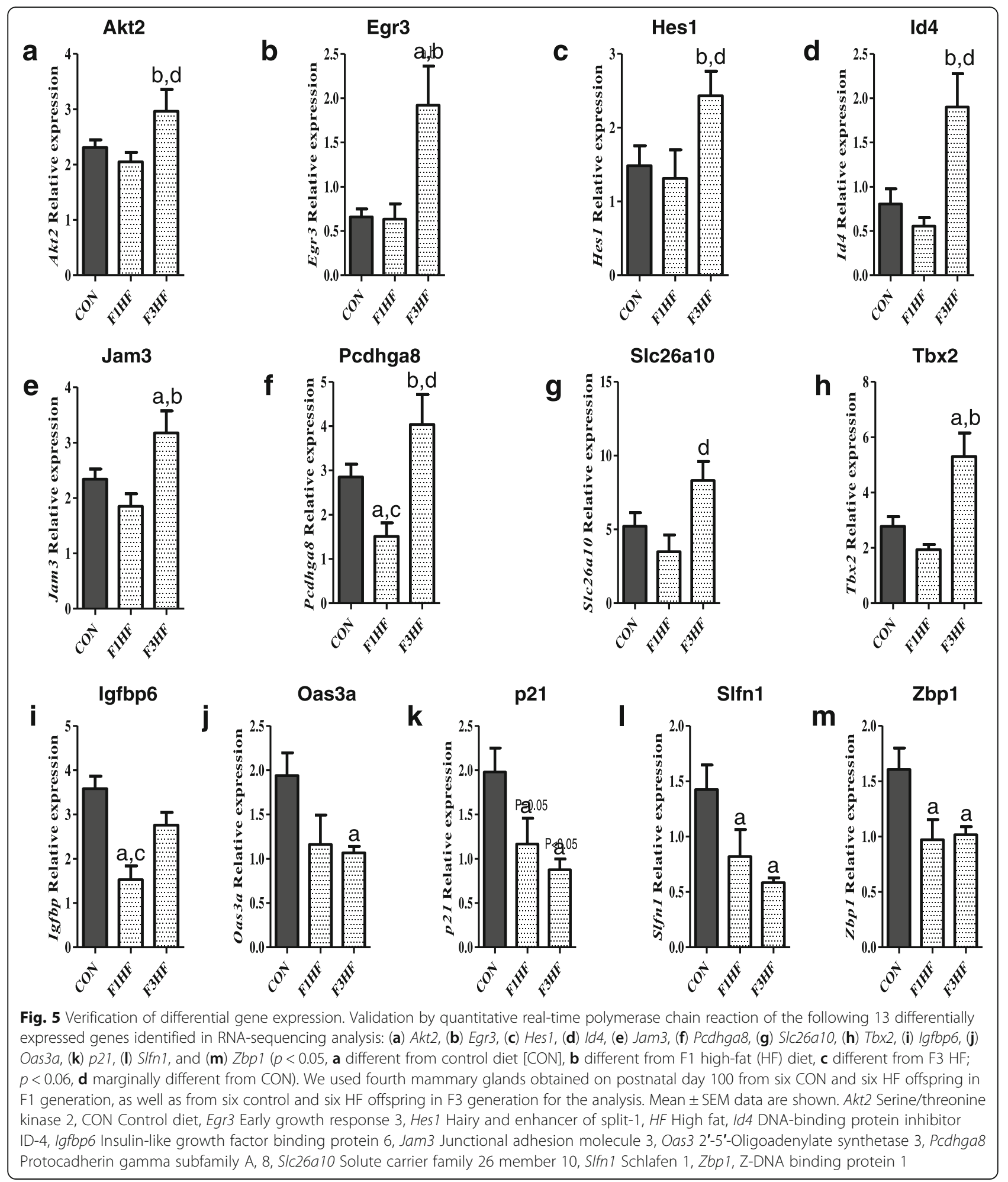

methylation patterns are erased in the zygote [17, 22, 23]. However, a growing number of studies indicate that some genes in preimplantation zygotes can escape the complete loss of methylation marks that were established during reprogramming events of germ cells [32, 33]. Further, it has been shown that changes in histone marks in the preconception oocytes can be transmitted across generations [34]. Results of our previous studies, as well as results of multiple other studies, indicate that various maternal exposures after the first week of gestation, 
such as endocrine disruptors ethinylestradiol (EE2) [13], 2,3,7,8-tetrachlorodibenzo-p-dioxin (TCDD) [35], vinclozolin [19], or DDT in rats [20], or bisphenol A [36], cause transgenerational alterations seen in F3 generation offspring.

Why, then, is the F3 generation not affected [13] if the exposure starts before conception and involves both the preimplantation period and the period when PGCs travel to the genital ridge $[22,23]$ ? It is possible that although F1 generation PGCs are affected directly by the in utero HF environment in both our previous study and the present study, the changes can be transgenerationally inherited only if the somatic cells in the blastocyst giving rise to PGCs were not also affected. If true, maternal exposures that start before conception and continue through pregnancy can have multigenerational effects only involving the F1 and F2 generations, but not the F3 generation.

To determine if similar changes occur in gene transcription in F1 and F3 generations, when they are caused by a direct exposure in $\mathrm{F} 1$ generation or inherited in F3 generation through the germline, we performed RNAseq analysis using normal mammary glands unexposed to the carcinogen DMBA, which were obtained from 100-day-old offspring of HF and control diet-fed dams. Surprisingly, over three times more DEGs were seen in the F3 than in the F1 generation mammary glands. Other groups have also reported greater gene transcription differences in F3 than F1 generation offspring. Ma et al. [35] found that maternal exposure to TCDD between GDs 8 and 14 resulted in a greater increase in both messenger RNA and protein expression of the imprinted gene insulin-like growth factor 2 (IGF2) in the rat liver of F3 than in F1 offspring. The increase was not caused by differences in DNA methylation patterns, which appeared similar in F1 and F3 generation offspring [35]. Consistent with these data, the differences in DNA methylation that we found earlier in F1 and F3 generations between control and maternal EE2 exposure groups were similar [13]. Thus, mechanisms other than changes in DNA methylation likely explain the increase in DEGs in the F3 generation offspring compared with F1 generation offspring.

From among all the DEGs found in our RNA-seq analysis, we selected the ones that were similarly altered in both F1 and F3 generation offspring of dams fed a HF diet during pregnancy compared with their controls for further analysis, because there possibly exists a connection between these 48 DEGs and the transgenerational inheritance of increased breast cancer risk. According to IPA, top biological functions of the DEGs were (1) development (cellular, embryonic, organ, organismal, and tissue), (2) cellular functions (cell cycle, proliferation, and morphology), (3) cancer and tumor morphology, and (4) inflammatory response (Additional file 7: Table S5). Considering that the main component of the HF diet was CO, a source of $n-6$ PUFAs, it is not surprising to find inflammatory response on this list of affected biofunctions. n-6 PUFAs and their arachidonic acid-derived eicosanoids are considered proinflammatory and associated with many inflammatory diseases, such as various types of cancer [11].

Five top upstream regulators of the 48 DEGs were DLL3 and JAG1 (Notch ligands) [37]; MSGN1 (transcriptional activator of a Notch signaling program) [32]; and $I R F 3$ and IRF7, which are both key transcriptional regulators of interferons and macrophages [30]. Notch signaling may also regulate IRF activity [38]. These results suggest that Notch signaling may be altered in the offspring by maternal HF intake. Notch signaling was also among the five top pathways altered in the offspring of HF diet-fed dams, in addition to VDR/RXR and FXR/ RXR activation, hereditary breast cancer signaling, and PTEN signaling. Researchers in earlier studies have found that maternal HF diet intake increases offspring Notch signaling in the hippocampus [39] and neural stem cells in mice [40]. Because the Notch pathway regulates stem cell maintenance, cell fate specification, differentiation, proliferation, motility, and survival during embryonic development and in cancers [41], our findings indicate that upregulation of Notch signaling in the normal mammary glands in F1 and F3 generation offspring of HF diet-fed dams may contribute to their increased mammary cancer risk.

To further characterize the functional roles of the DEGs, the networks hosted by these genes were constructed using KDDN analysis [29]. KDDN analysis identified differential connections among transcription factors that exist only in the mammary glands of offspring of HF diet-fed dams or only in the control offspring. In the HF group, genes identified as connected to the node genes were related to poor prognosis (SEMA5B, ID4, TBX2, GRHL3, DPF3), increased cellular proliferation and migration (GRHL3, PARP8, JAM3), and altered immune response (ZBP1, EGR3). As an example, the KDDN analysis indicated that upregulated grainyhead like transcription factor 3 (GRHL3) interacted with inhibitor of DNA binding 4 (ID4) and insulin-like growth factor binding protein 6 (IGFBP6) in the HF group, whereas its downregulation in the control group interacted with glycerophosphocholine phosphodiesterase 1 (GPCPD1) and small nucleolar RNA, H/ACA Box 41 (SNORA41). Upregulation of GRHL3 is strongly implicated in breast cancer [42], possibly by increasing the epithelial-mesenchymal transition [43]. ID4, upregulated in the offspring of HF diet-fed dams, is associated with poor prognosis of breast cancer, inhibits $B R C A 1$ function in basal-like breast cancer [44], and promotes 
chemoresistance [45]. IGFBP6 was downregulated in HF offspring, and it acts as a tumor suppressor [46]. The interactions of downregulated GRHL3 in the control offspring with downregulated GPCPD1 and SNORA41 are indicative of good cancer prognosis [47, 48]. Changes in the expression of these genes and their interaction with each other suggest that maternal HF n-6 PUFA diet may not only lead to a transgenerational increase in breast cancer risk but also increase breast cancer mortality. This conclusion is consistent with our recent finding that maternal EE2 exposure during pregnancy increased resistance to antiestrogen therapy of $\mathrm{ER}^{+}$mammary tumors in the offspring [49].

\section{Conclusions}

Our findings indicate that consuming a HF n-6 PUFA diet between GDs 10 and 20 during pregnancy causes a transgenerational increase in mammary cancer risk in mice. We also observed over three times more changes in the mammary gland transcriptome in F3 than in F1 generation offspring of HF diet-fed dams, suggesting that germline inheritance of increased mammary cancer risk may involve additional pathways to those altered in the adult mammary gland by a direct exposure of the fetal somatic cells.

\section{Additional files}

Additional file 1: Table S1. Nutritional content of control (modified AIN93-G) and high-fat n-6 PUFA diets fed to pregnant mouse dams. (DOCX $15 \mathrm{~kb}$ )

Additional file 2: Table S2. Primer sequences used in this study. (DOCX $77 \mathrm{~kb})$

Additional file 3: Figure S1. Tumor histopathology of all tumors collected from F1 and F3 generation offspring of dams exposed to either control (CON) or high-fat (HF) diet during pregnancy. a Tumor status of CON offspring. b Tumor status of HF offspring. (PDF $129 \mathrm{~kb}$ )

Additional file 4: Figure S2. Mammary tumor multiplicity was not altered between (a) F1 control (CON; $n=30$ mice) and high-fat (HF; $n=29$ mice) offspring or (b) F3 CON ( $n=19$ mice) and HF ( $n=24$ mice) generation offspring. (PDF $54 \mathrm{~kb}$ )

Additional file 5: Table S3. Common differentially expressed genes in the mammary glands of F1 and F3 generation offspring of dams fed high-fat n-6 PUFA diet during pregnancy, compared with control mice. (DOCX $104 \mathrm{~kb}$ )

Additional file 6: Table S4. Top differentially expressed pathways and predicted upstream regulators in F1 and F3 offspring of control or highfat fed dams, identified in Ingenuity Pathway Analysis. (DOCX $47 \mathrm{~kb}$ )

Additional file 7: Table S5. Top differentially expressed diseases and biofunctions between F1 and F3 offspring of dams fed control or high-fat diet during pregnancy, identified using Ingenuity Pathway Analysis. (DOCX $65 \mathrm{~kb}$ )

Additional file 8: Supplementary reference list for KDDN. (DOCX $102 \mathrm{~kb}$ )

\section{Abbreviations}

AKR1C14: Aldo-keto reductase family 1, member C14; AKT2: Serine/threonine kinase 2; ALG6: a-1,3-Glucosyltransferase; ANKEF1: Ankyrin repeat and EFhand domain containing 1; ANOVA: Analysis of variance; CDNA: Complementary
DNA; CO: Corn oil; CON: Control; DDT: Dichlorodiphenyltrichloroethane; DEG: Differentially expressed gene; DLL3: Delta like canonical Notch ligand 3; DMBA: 7,12-Dimethylbenz[a]anthracene; DPF3: Double PHD fingers 3; E2: 17- $\beta$ Estradiol; EE2: Ethinylestradiol; EGR3: Early growth response 3; ER: Estrogen receptor; FXR/RXR: Farnesoid $X$ receptor/retinoid $X$ receptor; GAPDH: Glyceraldehyde 3-phosphate dehydrogenase; GD: Gestational day; GPCPD1: Glycerophosphocholine phosphodiesterase 1; GPCR: G proteincoupled receptor; GRHL3: Grainyhead like transcription factor 3;

GWAS: Genome-wide association study; HES1: Hairy and enhancer of split-1; HF: High fat; ID4: DNA-binding protein inhibitor ID-4; IGF-1: Insulin-like growth factor 1; IGF2: Insulin-like growth factor 2; IGFBP6: Insulin-like growth factor binding protein 6; IPA: Ingenuity Pathway Analysis; IRF3: Interferon regulatory factor 3; IRF7: Interferon regulatory factor 7; JAG1: Jagged 1; JAM3: Junctional adhesion molecule 3; KDDN: Knowledge-fused differential dependency network: MAGIX: MAGI family member, X-linked; MPA: Medroxyprogesterone acetate; MSGN1: Mesogenin 1; MT-TS2: Mitochondrially encoded transfer RNA serine 2 (AGU/C); OAS3: 2'-5'-Oligoadenylate synthetase 3; OPTC: Opticin; P21: Cyclin-dependent kinase inhibitor 1; PCDHGA8: Protocadherin gamma subfamily A, 8; PGC: Primordial germ cell; PND: Postnatal day;

PTEN: Phosphatase and tensin homolog; PUFA: Polyunsaturated fatty acid; qPCR: Quantitative polymerase chain reaction; qRT-PCR: Quantitative real-time polymerase chain reaction; RNA-seq: RNA sequencing; SBO: Soybean oil; SEMA5B: Semaphorin 5B; SFA: Saturated fatty acid; SLC26A10: Solute carrier family 26 member 10; SLC26A3: Solute carrier family 26 member 3;

SLC5A3: Solute carrier family 5 member 3; SLC6A2: Solute carrier family 6 member 2; SLFN1: Schlafen 1; SNORA41: Small nucleolar RNA, H/ACA box 41; SNP: Single-nucleotide polymorphism; STAT5: Signal transducer and activator of transcription 5; TBX2: T-box 2; TCDD: 2,3,7,8-tetrachlorodibenzo-p-dioxin; TEB: Terminal end bud; VDR/RXR: Vitamin D receptor/retinoid $X$ receptor; ZBP1: Z-DNA binding protein 1; ZFP683: Zinc finger protein 683

\section{Acknowledgements}

We thank Samuel Wopperer, Helen Jung and Catherine Sevigny for their excellent technical assistance.

\section{Funding}

This work was supported by National Institutes of Health grants U54 CA149147 and R01 CA164384 (to LHC) and P30 CA51008 (to the Lombardi Comprehensive Cancer Center; funding for shared resources).

\section{Availability of data and materials}

The datasets supporting the conclusions of this study are included within the article and its additional files.

\section{Authors' contributions}

LHC is the principal investigator of the study and study supervisor. LHC, SdA, and NMN conceptualized and designed the transgenerational study. NMN and LHC wrote and reviewed the manuscript. NMN and LHC developed the methodology for the breeding and treatment of the animals. NMN, MIC, CB, $\mathrm{XZ}, \mathrm{BW}$, and $\mathrm{CY}$ were responsible for all aspects of the study concerning the animal model, including breeding, treatment, diet, collection of tissues, and tissue postprocessing. $\mathrm{L}, \mathrm{XW}$, and JX performed the RNA-seq analysis and assisted in the interpretation of the data along with NMN and LHC. FdOA performed the validation GRT-PCR of the RNA-seq. MM performed the analysis of the mammary gland morphology. All authors read and approved the final manuscript.

\section{Competing interests}

The authors declare that they have no competing interests.

Ethics approval

All animal procedures were approved by the Georgetown University Animal Care and Use Committee (GUACUC).

\section{Publisher's Note}

Springer Nature remains neutral with regard to jurisdictional claims in published maps and institutional affiliations.

\section{Author details}

'Department of Oncology, Georgetown University, Research Building, Room E407, 3970 Reservoir Road, NW, Washington, DC 20057, USA. ${ }^{2}$ UTHealth 
Graduate School of Biomedical Sciences, The University of Texas MD Anderson Cancer Center, Houston, TX, USA. ${ }^{3}$ Department of Electrical and Computer Engineering, Virginia Tech, Arlington, VA, USA.

Received: 16 December 2016 Accepted: 7 June 2017

Published online: 03 July 2017

\section{References}

1. Ferlay J, Soerjomataram I, Dikshit R, Eser S, Mathers C, Rebelo M, et al. Cancer incidence and mortality worldwide: sources, methods and major patterns in GLOBOCAN 2012. Int J Cancer. 2015:136:E359-86.

2. Antoniou AC, Easton DF. Models of genetic susceptibility to breast cancer. Oncogene. 2006;25:5898-905

3. Oldenburg RA, Meijers-Heijboer H, Cornelisse CJ, Devilee P. Genetic susceptibility for breast cancer: how many more genes to be found? Crit Rev Oncol Hematol. 2007;63:125-49.

4. Albuquerque RC, Baltar VT, Marchioni DM. Breast cancer and dietary patterns: a systematic review. Nutr Rev. 2014;72:1-17.

5. Walker $\mathrm{CL}$, Ho SM. Developmental reprogramming of cancer susceptibility. Nat Rev Cancer. 2012;12:479-86.

6. Blasbalg TL, Hibbeln JR, Ramsden CE, Majchrzak SF, Rawlings RR. Changes in consumption of omega-3 and omega- 6 fatty acids in the United States during the 20th century. Am J Clin Nutr. 2011;93:950-62.

7. Cordain L, Eaton SB, Sebastian A, Mann N, Lindeberg S, Watkins BA, et al. Origins and evolution of the Western diet: health implications for the $21 \mathrm{st}$ century. Am J Clin Nutr. 2005:81:341-54.

8. Guyenet SJ, Carlson SE. Increase in adipose tissue linoleic acid of US adults in the last half century. Adv Nutr. 2015;6:660-4.

9. Hilakivi-Clarke L, Clarke R, Onojafe I, Raygada M, Cho E, Lippman ME. A maternal diet high in n-6 polyunsaturated fats alters mammary gland development, puberty onset, and breast cancer risk among female rat offspring. Proc Natl Acad Sci U S A. 1997;94:9372-7.

10. Hilakivi-Clarke L, Onojafe I, Raygada M, Cho E, Clarke R, Lippman M. Breast cancer risk in rats fed a diet high in n-6 polyunsaturated fatty acids during pregnancy. J Natl Cancer Inst. 1996:88:1821-7.

11. Marion-Letellier R, Savoye G, Ghosh S. Polyunsaturated fatty acids and inflammation. IUBMB Life. 2015;67:659-67.

12. Harizi H, Corcuff JB, Gualde N. Arachidonic-acid-derived eicosanoids: roles in biology and immunopathology. Trends Mol Med. 2008;14:461-9.

13. de Assis S, Warri A, Cruz MI, Laja O, Tian Y, Zhang B, et al. High-fat or ethinyl-oestradiol intake during pregnancy increases mammary cancer risk in several generations of offspring. Nat Commun. 2012;3:1053.

14. Stark AH, Kossoy G, Zusman I, Yarden G, Madar Z. Olive oil consumption during pregnancy and lactation in rats influences mammary cancer development in female offspring. Nutr Cancer. 2003;46:59-65.

15. Walker BE. Tumors in female offspring of control and diethylstilbestrol-exposed mice fed high-fat diets. J Nat Cancer Inst. 1990;82:50-4.

16. Luijten M, Thomsen AR, van den Berg JA, Wester PW, Verhoef A, Nagelkerke $\mathrm{NJ}$, et al. Effects of soy-derived isoflavones and a high-fat diet on spontaneous mammary tumor development in Tg.NK (MMTV/c-neu) mice. Nutr Cancer. 2004:50:46-54

17. Heard E, Martienssen RA. Transgenerational epigenetic inheritance: myths and mechanisms. Cell. 2014;157:95-109.

18. Skinner MK. What is an epigenetic transgenerational phenotype? F3 or F2. Reprod Toxicol. 2008;25:2-6.

19. Anway MD, Leathers C, Skinner MK. Endocrine disruptor vinclozolin induced epigenetic transgenerational adult-onset disease. Endocrinology. 2006;147:5515-23.

20. Skinner MK, Manikkam M, Tracey R, Guerrero-Bosagna C, Haque M, Nilsson EE. Ancestral dichlorodiphenyltrichloroethane (DDT) exposure promotes epigenetic transgenerational inheritance of obesity. BMC Med. 2013;11:228.

21. Choi CS, Gonzales EL, Kim KC, Yang SM, Kim JW, Mabunga DF, et al. The transgenerational inheritance of autism-like phenotypes in mice exposed to valproic acid during pregnancy. Sci Rep. 2016;6:36250.

22. Seisenberger S, Andrews S, Krueger F, Arand J, Walter J, Santos F, et al. The dynamics of genome-wide DNA methylation reprogramming in mouse primordial germ cells. Mol Cell. 2012;48:849-62.

23. Skinner MK, Haque CG, Nilsson E, Bhandari R, McCarrey JR. Environmentally induced transgenerational epigenetic reprogramming of primordial germ cells and the subsequent germ line. PLoS One. 2013;8:e66318.
24. Liu FL, Zhang YM, Pares GV, Reidy KC, Zhao WZ, Zhao A, et al. Nutrient intakes of pregnant women and their associated factors in eight cities of China: a cross-sectional study. Chin Med J (Engl). 2015;128:1778-86.

25. Bosaeus M, Hussain A, Karlsson T, Andersson L, Hulthen L, Svelander C, et al. A randomized longitudinal dietary intervention study during pregnancy: effects on fish intake, phospholipids, and body composition. Nutr J. 2015;14:1

26. de Assis S, Warri A, Cruz MI, Hilakivi-Clarke L. Changes in mammary gland morphology and breast cancer risk in rats. J Vis Exp. 2010;44:e2260.

27. Russo $\mathrm{H}$, Russo J. Mammary gland neoplasia in long-term rodent studies. Environ Health Perspect. 1996;104:938-67.

28. Russo J, Hu YF, Yang X, Russo $\|_{\text {. }}$. Developmental, cellular, and molecular basis of human breast cancer. J Natl Cancer Inst Monogr. 2000;27:17-37.

29. Tian Y, Zhang B, Hoffman EP, Clarke R, Zhang Z, Shih I, et al. KDDN: an open-source Cytoscape app for constructing differential dependency networks with significant rewiring. Bioinformatics. 2015;31:287-9.

30. Gonzalez MJ, Ruiz-Garcia A, Monsalve EM, Sanchez-Prieto R, LaBorda J, Diaz-Guerra MJ, et al. DLK1 is a novel inflammatory inhibitor which interferes with NOTCH1 signaling in TLR-activated murine macrophages. Eur J Immunol. 2015;45:2615-27.

31. Penton AL, Leonard LD, Spinner NB. Notch signaling in human development and disease. Semin Cell Dev Biol. 2012;23:450-7.

32. Chalamalasetty RB, Dunty Jr WC, Biris KK, Ajima R, lacovino M, Beisaw A, et al. The Wnt3a/B-catenin target gene Mesogenin 1 controls the segmentation clock by activating a Notch signalling program. Nat Commun. 2011:2:390

33. Orozco LD, Rubbi L, Martin LJ, Fang F, Hormozdiari F, Che N, et al. Intergenerational genomic DNA methylation patterns in mouse hybrid strains. Genome Biol. 2014;15:R68.

34. Gaydos L, Wang W, Strome S. Gene repression. H3K27me and PRC2 transmit a memory of repression across generations and during development. Science. 2014;345:1515-8.

35. Ma J, Chen X, Liu Y, Xie Q, Sun Y, Chen J, et al. Ancestral TCDD exposure promotes epigenetic transgenerational inheritance of imprinted gene Igf2: Methylation status and DNMTs. Toxicol Appl Pharmacol. 2015:289:193-202

36. Ziv-Gal A, Wang W, Zhou C, Flaws JA. The effects of in utero bisphenol A exposure on reproductive capacity in several generations of mice. Toxicol Appl Pharmacol. 2015;284:354-62.

37. Murata A, Yoshino M, Hikosaka M, Okuyama K, Zhou L, Sakano S, et al. An evolutionary-conserved function of mammalian notch family members as cell adhesion molecules. PLoS One. 2014;9:e108535.

38. Restivo G, Nguyen BC, Dziunycz P, Ristorcelli E, Ryan RJ, Özuysal ÖY, et al. IRF6 is a mediator of Notch pro-differentiation and tumour suppressive function in keratinocytes. EMBO J. 2011;30:4571-85.

39. Mendes-da-Silva C, Lemes SF, Baliani TS, Versutti MD, Torsoni MA. Increased expression of Hes 5 protein in Notch signaling pathway in the hippocampus of mice offspring of dams fed a high-fat diet during pregnancy and suckling. Int J Dev Neurosci. 2015:40:35-42.

40. Yu M, Jiang M, Yang C, Wu Y, Liu Y, Cui Y, et al. Maternal high-fat diet affects Msi/Notch/Hes signaling in neural stem cells of offspring mice. J Nutr Biochem. 2014;25:227-31.

41. Harrison $H$, Farnie G, Brennan KR, Clarke RB. Breast cancer stem cells: something out of notching? Cancer Res. 2010;70:8973-6.

42. Xu H, Liu C, Zhao Z, Gao N, Chen G, Wang Y, et al. Clinical implications of GRHL3 protein expression in breast cancer. Tumour Biol. 2014;35:1827-31.

43. Zhao P, Guo S, Tu Z, Di L, Zha X, Zhou H, et al. Grhl3 induces human epithelial tumor cell migration and invasion via downregulation of $\mathrm{E}$ cadherin. Acta Biochim Biophys Sin Shanghai. 2016;48:266-74.

44. Baker LA, Holliday H, Swarbrick A. ID4 controls luminal lineage commitment in normal mammary epithelium and inhibits BRCA1 function in basal-like breast cancer. Endocr Relat Cancer. 2016:23:R381-92.

45. Qi K, Li Y, Li X, Lei X, Wang B, Zhang L, et al. Id4 promotes cisplatin resistance in lung cancer through the p38 MAPK pathway. Anticancer Drugs. 2016;27:970-8

46. Kaulsay KK, Ng EH, Ji CY, Ho GH, Aw TC, Lee KO. Serum IGF-binding protein-6 and prostate specific antigen in breast cancer. Eur J Endocrinol. 1999:140:164-8.

47. Lesjak MS, Marchan R, Stewart JD, Rempel E, Rahnenfuhrer J, Hengstler JG. EDI3 links choline metabolism to integrin expression, cell adhesion and spreading. Cell Adh Migr. 2014;8:499-508. 
48. Bao L, Zhang Y, Wang J, Wang H, Dong N, Su X, et al. Variations of chromosome 2 gene expressions among patients with lung cancer or non-cancer. Cell Biol Toxicol. 2016;32:419-35.

49. Hilakivi-Clarke L, Wärri AM, Bouker KB, Zhang X, Cook KL, Jin L, et al. Effects of in utero exposure to ethinyl estradiol on tamoxifen resistance and breast cancer recurrence in a preclinical model. J Nat Cancer Inst. 2016;109:djw188.

Submit your next manuscript to BioMed Central and we will help you at every step:

- We accept pre-submission inquiries

- Our selector tool helps you to find the most relevant journal

- We provide round the clock customer support

- Convenient online submission

- Thorough peer review

- Inclusion in PubMed and all major indexing services

- Maximum visibility for your research

Submit your manuscript at www.biomedcentral.com/submit 